
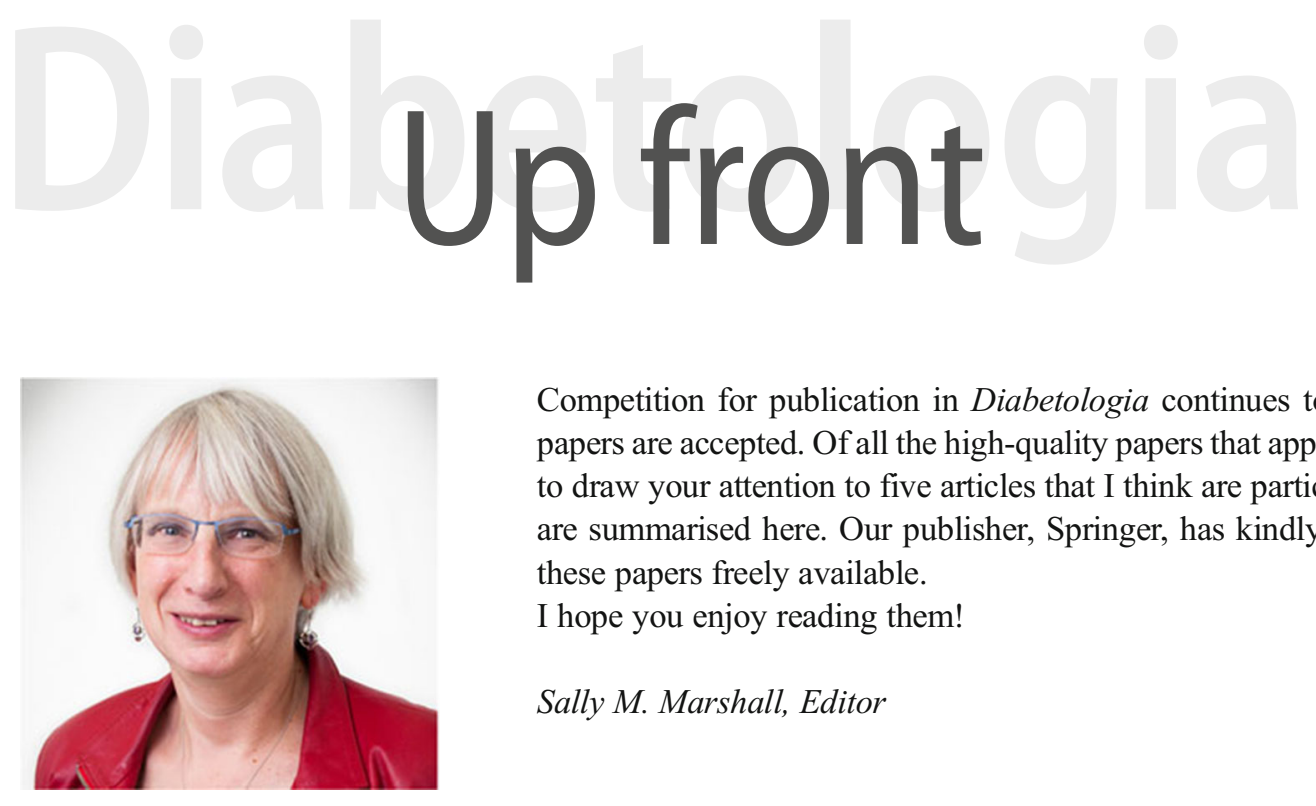

Competition for publication in Diabetologia continues to grow, and less than $20 \%$ of papers are accepted. Of all the high-quality papers that appear in this month's issue I want to draw your attention to five articles that I think are particularly interesting. The articles are summarised here. Our publisher, Springer, has kindly made the full text of each of these papers freely available.

I hope you enjoy reading them!

Sally M. Marshall, Editor

\section{Real-world evidence: the devil is in the detail}

Mugdha Gokhale, Til Stürmer, John B. Buse

The healthcare community is abuzz with discussions about real world evidence (RWE), which provides evidence regarding the utilisation and potential benefits and harms of healthcare interventions, based on data from routine clinical practice. While the reflection of diverse real-world patterns increases the attractiveness of RWE, it also increases the chance of several biases in real-world studies. In this issue (https://doi.org/10.1007/s00125-020-05217-1), Gokhale et al summarise key elements in RWE studies, including commonly encountered issues and methods to address these. While there is no one-size-fits-all solution to designing RWE studies, the authors explain that critical elements include a clear prespecified protocol with a well-defined research question, a fit-for-purpose data source, therapeutically equivalent treatments as comparators, robust state-of-the-art study design, analytical techniques to minimise biases, and clearly reported methods and results per published guidelines. The review also points to some newer areas of application of RWE that are relevant to diabetes research and beyond.

(D) The figures from this review are available as a downloadable slideset.
Using genetics to decipher the link between type 2 diabetes and cancer: shared aetiology or downstream consequence?

Emma E. Vincent, Hanieh Yaghootkar

The global prevalence of type 2 diabetes is estimated to reach over 500 million by 2030 . To compound this health crisis, observational epidemiological studies have consistently reported that people with type 2 diabetes have a higher risk of certain types of cancer. To direct prevention and intervention strategies the association between the two disease states needs to be understood. In this issue, Vincent and Yaghootkar (https://doi.org/10.1007/s00125020-05228-y) review how large-scale genome-wide association studies (GWAS) have been employed to investigate a possible relationship between type 2 diabetes and cancer. The evidence for shared genetic aetiology between alleles predisposing to type 2 diabetes and the observationally associated cancers is explored. The authors also discuss how GWAS and techniques such as Mendelian randomisation are used to investigate whether type 2 diabetes causes cancer or whether this association is driven by a particular metabolic feature, such as elevated insulin levels, or by an associated trait, such as obesity. Finally, the authors discuss areas for future study, including genetics studies of type 2 diabetes and cancer progression.

(1) The figure from this review is available as a downloadable slideset. 
A systematic review of trends in all-cause mortality among people with diabetes

Lei Chen, Rakibul M. Islam, Joanna Wang, Thomas R. Hird, Meda E. Pavkov, Edward W. Gregg, Agus Salim, Maryam Tabesh, Digsu N. Koye, Jessica L. Harding, Julian W. Sacre, Elizabeth L.M. Barr, Dianna J. Magliano, Jonathan E. Shaw

Some studies have reported falling mortality among populations with diabetes, but consistency of this trend among various populations with diabetes is unclear. In this issue, Chen and colleagues (https://doi.org/10.1007/s00125-020-05199-0) report a systematic review of 35 studies on the trends in allcause mortality in people with diabetes from 1970 to 2016. They found that all-cause mortality has declined in nearly $80 \%$ of predominantly Europid populations with diabetes from 2000 to 2016 . Furthermore, for nearly $60 \%$ of the populations, the reduction in mortality seen among those with diabetes was greater than or similar to the reductions observed in those without diabetes. However, there was under-representation of younger age groups and non-Europid populations in the published literature, and lack of mortality data from low- and middleincome countries. Thus, patterns of diabetes mortality remain uncertain in these populations. These findings highlight the significance of maintaining and improving cardiometabolic management in diabetes in order to achieve ongoing reductions in mortality in people with diabetes.

Live-born children after assisted reproduction in women with type 1 diabetes and type 2 diabetes: a nationwide cohort study

Michael Due Larsen, Dorte Møller Jensen, Jens Fedder, Line Riis Jølving, Bente Mertz Nørgård

Type 1 and type 2 diabetes are among the most common chronic diseases in women during the fertile years and previous studies have indicated that women with diabetes have difficulty conceiving compared with reference populations. However, the efficacy of assisted reproductive technology (ART) in women with diabetes has never been studied. In this issue, Larsen and colleagues (https://doi.org/10.1007/s00125-020-05193-6) report the results of a nationwide study of 594 women with either type 1 or type 2 diabetes in the Danish ART Registry, between 2006 and 2017. The authors found that women with type 2 diabetes have a decreased chance of live birth per embryo transfer compared with women without diabetes. Furthermore, the results suggested that the decrease is related to a lower chance of implantation of the embryo. Women with type 1 diabetes had an equivalent chance of a live birth per embryo transfer as women without diabetes. The findings in women with type 2 diabetes did not seem to be driven by obesity as the same pattern was seen in both normal-weight and obese women. The authors conclude that their findings should provide reassurance for women with type 1 diabetes considering ART treatment but that prospective studies need to be performed to confirm the findings for women with types 2 diabetes in other settings and to investigate the underlying mechanisms.

Leptin production capacity determines food intake and susceptibility to obesity-induced diabetes in OikawaNagao Diabetes-Prone and Diabetes-Resistant mice

Akira Asai, Mototsugu Nagao, Koji Hayakawa, Teruo Miyazawa, Hitoshi Sugihara, Shinichi Oikawa

Even in the modern 'obesogenic' environment of plentiful food supply, not all people become obese by overeating. However, it remains poorly understood what determines individual differences in spontaneous meal size. In this issue, Asai and Nagao et al (https://doi.org/10.1007/s00125-020-05191-8) investigate the potential role of leptin production capacity in determining spontaneous meal size and consequent susceptibility to obesityinduced diabetes using selectively bred Oikawa-Nagao Diabetes-Prone (ON-DP) and Diabetes-Resistant (ON-DR) mice. The authors demonstrate that, prior to the development of obesity-induced diabetes, the spontaneously hyperphagic ON-DP mice had a lower circulating leptin level than the control ON-DR mice. ON-DP mice had a lower leptin production capacity in adipose tissue, probably because of the higher DNA methylation level in the Lep promoter region compared with that in ON-DR mice. The authors conclude that leptin production capacity, especially before the development of obesity, may have diagnostic potential for predicting individual risk of obesity and future onset of type 2 diabetes.

All text supplied by the authors. 